Article

\title{
Effects of Antagonists on Mycotoxins of Seedborne Fusarium spp. in Sweet Corn
}

\author{
Mary E. Ridout ${ }^{1, *}$, Bruce Godfrey ${ }^{2}$ and George Newcombe ${ }^{3}[$ \\ 1 University of Idaho Extension, Washington County, College of Agriculture and life Sciences, Weiser, \\ ID 83672, USA \\ 2 Department of Civil \& Environmental Engineering, University of Washington, Seattle, WA 98105, USA \\ 3 Department of Forest, Rangelands and Fire Sciences, University of Idaho, Moscow, ID 83844, USA \\ * Correspondence: mridout@uidaho.edu
}

Received: 3 July 2019; Accepted: 23 July 2019; Published: 25 July 2019

\begin{abstract}
Fusarium species coexist as toxigenic, systemic pathogens in sweet corn seed production in southwestern Idaho, USA. We hypothesized that fungal antagonists of seedborne Fusarium would differentially alter production of Fusarium mycotoxins directly and/or systemically. We challenged the Fusarium complex by in vitro antagonism trials and in situ silk and seed inoculations with fungal antagonists. Fungal antagonists reduced growth and sporulation of Fusarium species in vitro from $40.5 \%$ to as much as $100 \%$. Pichia membranifaciens and Penicillium griseolum reduced fumonisin production by $F$. verticillioides by $73 \%$ and $49 \%$, respectively, while $P$. membranifaciens and a novel Penicillium sp. (WPT) reduced fumonisins by F. proliferatum $56 \%$ and $78 \%$, respectively. In situ, pre-planting inoculation of seeds with Penicillium WPT systemically increased fumonisins in the resulting crop. Morchella snyderi applied to silks of an F1 cross systemically reduced deoxynivalenol by $47 \%$ in mature seeds of the F2. Antagonists failed to suppress Fusarium in mature kernels following silk inoculations, although the ratio of F. verticillioides to total Fusarium double with some inoculants. Fusarium mycotoxin concentrations in sweet corn seed change systemically, as well as locally, in response to the presence of fungal antagonists, although in Fusarium presence in situ was not changed.
\end{abstract}

Keywords: Fusarium verticillioides; Fusarium proliferatum; fumonisin; deoxynivalenol; fungal antagonists

Key Contribution: We identify fungal antagonists of the Fusarium complex in sweet corn seed production that suppress and stimulate mycotoxin production.

\section{Introduction}

Fungi produce many biochemicals through metabolic processes and in response to stimuli [1]. In fact, many fungi in the plant microbiome engage in "biochemical warfare" among themselves and with other microbes in the community [2]. They produce numerous defensive compounds to compete against other microbes and defend their space and resources [3]. Many of the compounds have been exploited by humans for antibiotic activity [4]; others pose a threat as "mycotoxins", metabolites with toxic effects on mammals [5].

Fusarium verticillioides, F. proliferatum, and F. graminearum are major maize pathogens and produce several mycotoxins [6]. Fusarium poae is a common weak pathogen in maize but also produces a number of toxic metabolites [7]. The former two produce fumonisins, while the latter two produce deoxynivalenol (DON). Both toxins are regulated by the US Food and Drug Administration [8,9] and may travel systemically throughout the plant beyond the Fusarium-infected tissues [10,11]. Although neither mycotoxin group is necessary to the Fusarium species for infection and disease development in maize [12], their production has been correlated with increased severity of disease $[13,14]$ and genes for 
pathogenicity have been found to be linked to mycotoxin biosynthesis [15-17]. These metabolites also have been observed to interact with host resistance genes [18] and facilitate infection of the host $[12,13]$. However, production of toxins can be specific to the pathogen strain, not just species, $[7,19,20]$, a behavior frequently obfuscating the exact functions of these biochemicals [21].

Other microbes are known to antagonize or interact with Fusarium in the ear microbiome and root systems of maize [22-24]. Such microbial competition can effect changes in Fusarium mycotoxin production [25-29]. In fact, Keyser et al. [30] postulated that fumonisins could function as defense compounds when they found that fumonisin extracts reduced growth of several Fusarium competitors from the maize ear microbiome. Bacon et al. [26,31] similarly found that $F$. verticilliodes produced fusaric acid toxic to the Fusarium biocontrol agent Bacillus mojavensis when challenged with the bacterium. Conversely, Yates et al. [32] found that Trichoderma viride could reduce fumonisin production by F. verticillioides. Similarly, Chatterjee et al. [33] found that Clonostachys rosea inhibited fumonisin synthesis by F. verticillioides but did not degrade the toxin. Hebbar et al. [34] identified a number of bacteria, including several Pseudomonas species, in maize plants producing antifungal metabolites inhibitory to Fusarium verticillioides. In other cases, effects were more complex [32].

Fusarium species themselves interact chemically within the complex. More than one Fusarium species may colonize a host [35] and some, like Fusarium verticillioides, may colonize the host asymptomatically [36,37]—all producing a variety of toxins. For instance, Fusarium graminearum and F. verticillioides are known to interact significantly both in laboratory antagonism assays and in the field in terms of both growth and toxin production $[35,38,39]$. However, the direction of the interaction (antagonistic or synergistic) is highly dependent on environmental parameters [21,38,39].

The southern region of Idaho, USA produces $70 \%$ of the world's sweet corn (Zea mays convar. saccharata var. rugosa) seed [40]. Here in the semi-arid, continental climate and alkaline soils [41,42], Fusarium verticillioides and F. proliferatum co-occur in the Fusarium pathogen complex in sweet corn seed production [43,44]. Seed becomes infected via wind-driven and rain-splashed spores colonizing ears and re-infects successive crops systemically from infected seed surfaces and soil [45,46]. In addition to multiple Fusarium species, numerous other microbes colonize the seeds through the silks, some of which are antagonistic to the Fusarium species

Most frequently, attempts to understand the interactions of other microbes with toxigenic Fusarium species examine the interaction of a single antagonist with a single Fusarium species [24,27,28,47]. Yet, the complexity of the ear microbiome in maize $[22,23,27]$ indicates the biochemical behaviors may be much more complex as well. In a series of field trials, we surveyed the Fusarium pathogens in sweet corn fields in Nampa, ID, USA.

We hypothesized that different Fusarium species present during infection of the ears at silking would differentially alter mycotoxin production in response to antagonism by five fungal species with putative antagonism toward Fusarium species First, we confirmed antagonism of F. verticillioides and F. proliferatum by our putative antagonists and tested Fusarium isolates for fumonisin and DON production. To test our hypothesis, we quantified effects of fungal antagonists on sporulation of F. verticillioides and F. proliferatum and measured fumonisin production of both Fusarium when antagonized. We hypothesized that antagonists applied to ears at silking would induce changes in Fusarium mycotoxin production in situ, and that effects would occur locally in treated ears and systemically in plants grown from treated seed. We tested this hypothesis in 2015 field trials with the same suite of fungal antagonists. We inoculated emerging silks at flowering and seed at planting to compare local and systemic effects on mycotoxin content. To confirm systemic effects, we conducted a second year of field trials in 2016 and planted seeds developed from 2015 silk inoculations trials. Finally, we used quantitative polymerase chain reaction (qPCR) on mature seed from 2015 silk inoculation trials to determine the effects of fungal antagonists on Fusarium populations in the seed. 


\section{Results}

\subsection{Fusarium Species Present in Sweet Corn Seed from Nampa, Idaho, USA}

Four major Fusarium species were identified from the ears of sweet corn seed fields of Nampa, ID, USA: Fusarium verticillioides, F. proliferatum, F. temperatum [48] and F. poae. Fusarium proliferatum and F. verticillioides were most abundant in ears from 2014 and 2015 field trials (>95\% isolation frequency in non-sterilized seed). Fusarium temperatum occurred at relatively low frequency $(<30 \%)$ in both years. Only three isolates of F. poae were observed and only one was successfully isolated in the 2015 seed. As expected, when analyzed alone, only F. verticillioides and F. proliferatum produced detectible fumonisins. None of the isolates produced DON (data not shown). Fusarium proliferatum averaged $4 \mathrm{mg}$ of fumonisins per kilogram of fungal and substrate material whereas F. verticillioides averaged nearly twice that amount $(p<0.0001$, Figure 1$)$.

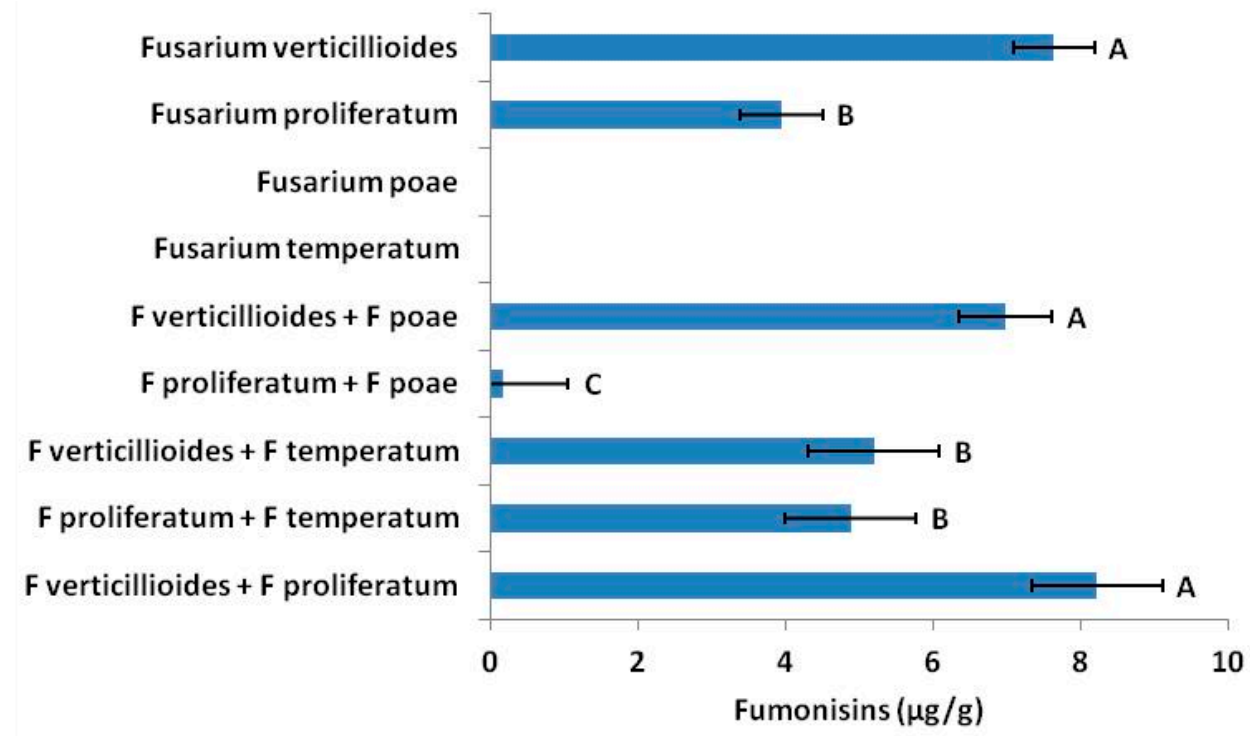

Figure 1. Co-occurring Fusarium species also altered fumonisin production of F. verticillioides and F. proliferatum in vitro. Although neither produced fumonisin, F. poae reduced fumonisin production by F. proliferatum by $96 \%$, and F. temperatum, reduced fumonisin by F. verticillioides by $32 \%$. Error bars indicate standard error $(\alpha=0.05)$.

\subsection{Antagonists of Sweet Corn Fusarium Species}

In laboratory antagonism assays, four fungal species isolated from native plants in Idaho, USA were found to strongly antagonize F. verticillioides and F. proliferatum. Penicillium sp. nov. WPT (hereafter Penicillium WPT), Penicillium griseolum, Clonostachys rosea, and Morchella snyderi inhibited growth and sporulation of both F. verticillioides and F. proliferatum. Clonostachys rosea also overgrew Fusarium species in vitro and sporulated on the surface of the parasitized Fusarium mycelia. Our strain of Pichia membranifaciens, which we isolated from immature sweet corn kernels from the field in 2014, inhibited growth of F. verticillioides and F. proliferatum in vitro as long as it remained viable. In broth culture, P. membranifaciens reduced the reproductive capacity of both F. proliferatum and F. verticillioides by suppressing conidia production by $99.9 \%$ compared to Fusarium-only controls (Figure 2a). Penicillium WPT, P. griseolum, M. snyderi, and C. rosea reduced sporulation of F. proliferatum by $67 \%, 71 \%, 73 \%$, and $87 \%(p<0.01)$, respectively (Figure 2a). Fusarium verticillioides was only slightly more resistant to filamentous antagonists with $M$. snyderi, P. griseolum, and C. rosea reducing sporulation by $40.5 \%$, $45 \%$, and $46 \%$, respectively $(p<0.05)$. However, Penicillium WPT failed to reduce sporulation of F. verticillioides $(p>0.05)$ (Figure $2 \mathrm{a})$. 

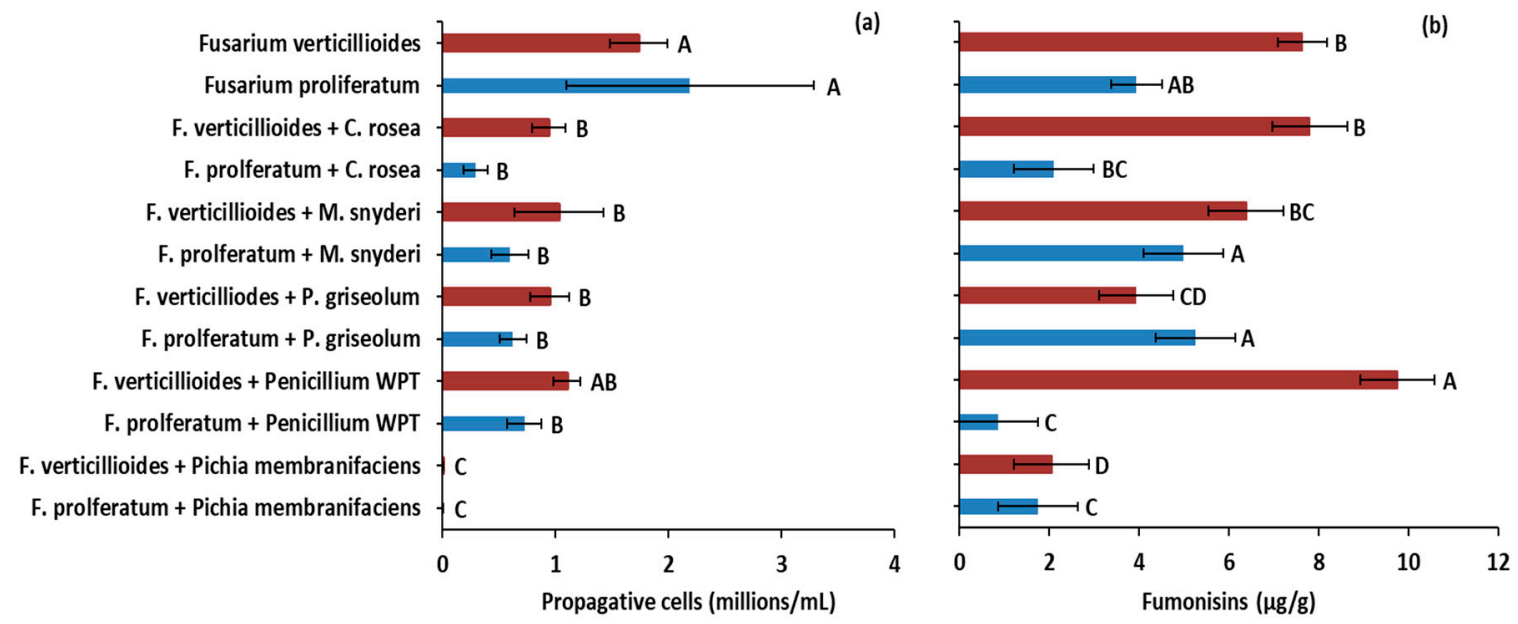

Figure 2. (a) Fungal antagonists of Fusarium species strongly reduced growth and sporulation of F. proliferatum and F. verticillioides in antagonism assays. Filamentous fungi antagonized F. proliferatum to a greater extent ( $67 \%$ to $87 \%$ reductions in sporulation) than F. verticillioides ( $37 \%$ to $46 \%$ reductions in sporulation). The yeast $P$. membranifaciens prevented development of both Fusarium species in broth culture. (b) However, antagonism had varying effects of fumonisin production that were specific to Fusarium sp. Both fumonisin producers antagonized by P. membranifaciens produced approximately $50 \%$ less fumonisin. When challenged with Penicillium WPT, F. proliferatum produced $78 \%$ less fumonisin while F. verticillioides produced $28 \%$ more. Antagonism with P. griseolum reduced fumonisin in F. verticillioides by $73 \%$ but had no effect on $F$. proliferatum. Error bars indicate standard error $(\alpha=0.05)$. Letters above red or blue bars indicate significant differences within a treatment set (F. verticillioides, red; F. proliferatum, blue).

\subsection{Antagonists Alter Fumonisin Production In Vitro}

In vitro, Fusarium antagonists significantly altered fumonisin production by F. verticillioides and F. proliferatum (Figure 2b). Fusarium verticillioides antagonized by P. griseolum and P. membranifaciens produced $49 \%(p=0.0003)$ and $73 \%(p<0.0001)$ less fumonisin, respectively, than it produced without antagonist pressure. Fusarium proliferatum challenged with P. membranifaciens and Penicillium WPT produced $56 \%(p=0.0396)$ and $78 \%$ ( $p=0.0040)$ less fumonisin, respectively, than when unchallenged. By contrast, when F. verticillioides was challenged with Penicillium WPT, the pathogen increased fumonisin production by nearly $28 \%$ ( $p=0.0359)$.

Although antagonists were effective at altering fumonisin expression by Fusarium species, co-occurring Fusarium species were equally effective (Figure 1). When F. proliferatum was challenged with co-occurring F. poae, it produced $96 \%(p=0.0005)$ less fumonisin than when unchallenged, and antagonism by F. temperatum reduced fumonisin production of F. verticillioides by $32 \%(p=0.0213)$.

\subsection{In Situ Antagonism is not Predicted In Vitro}

In vitro antagonism failed to predict effects in the field. In the 2015 silk inoculation trial, fumonisins were not reduced in ears treated with putative antagonists (Figure 3a). In fact, ears treated with Penicillium WPT at silking had nearly twice the fumonisin content of untreated ears from the control $(p=0.0026)$. DON production was low. However, treatments effects were still detected. DON was significantly higher $(p=0.0037)$ in seed from ears treated with most antagonists at silking, from a 34\% increase in seed inoculated with $M$. snyderi to $52 \%$ in seed treated with $M$. snyderi and P. membranifaciens combined (Figure 3b). Only C. rosea failed to increase DON in mature seed compared to untreated ears $(p=0.0737, \alpha=0.05)$. 

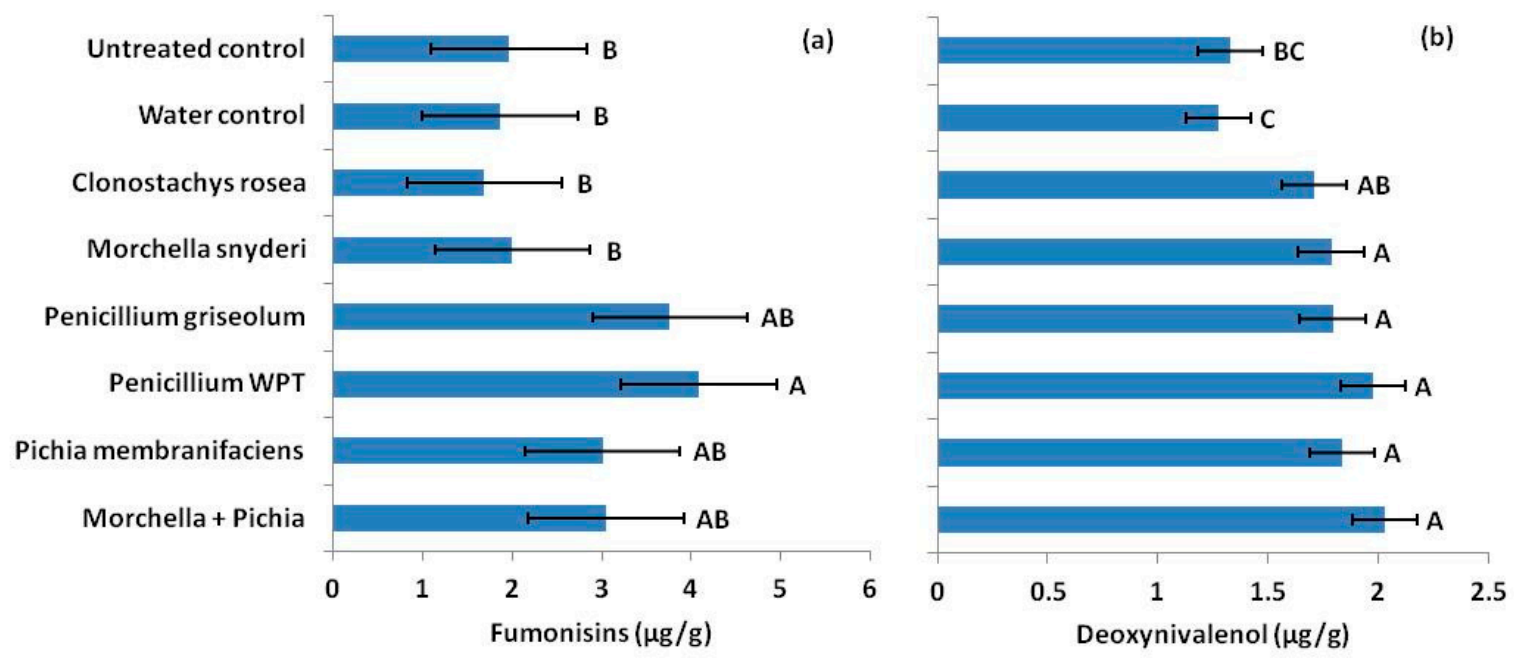

Figure 3. (a) In contrast to laboratory antagonism assays, fungal antagonists applied to developing ears at silking, mostly increased fumonisin concentrations in mature seed. In fact, Penicillium WPT nearly doubled fumonisins in treated ears compared to ears receiving no treatment (dry control). (b) Application of fungal antagonists also increased DON concentrations in mature seed compared to ears receiving no treatment (dry control) or ears that just receive distilled water application. Error bars indicate standard error $(\alpha=0.05)$.

\subsection{Putative Antagonists Act Systemically In Situ}

Effects of antagonists on mycotoxin production in sweet corn ears were also found to be systemic. When seeds were treated with antagonists at planting in the 2015 field trial, seed treatment with Penicillium WPT resulted in a five-fold increase in fumonisins ( $p=0.0217$, Figure 4$)$ compared to untreated controls but had no effect on DON ( $p>0.05$, data not shown) in mature seed from that planting.

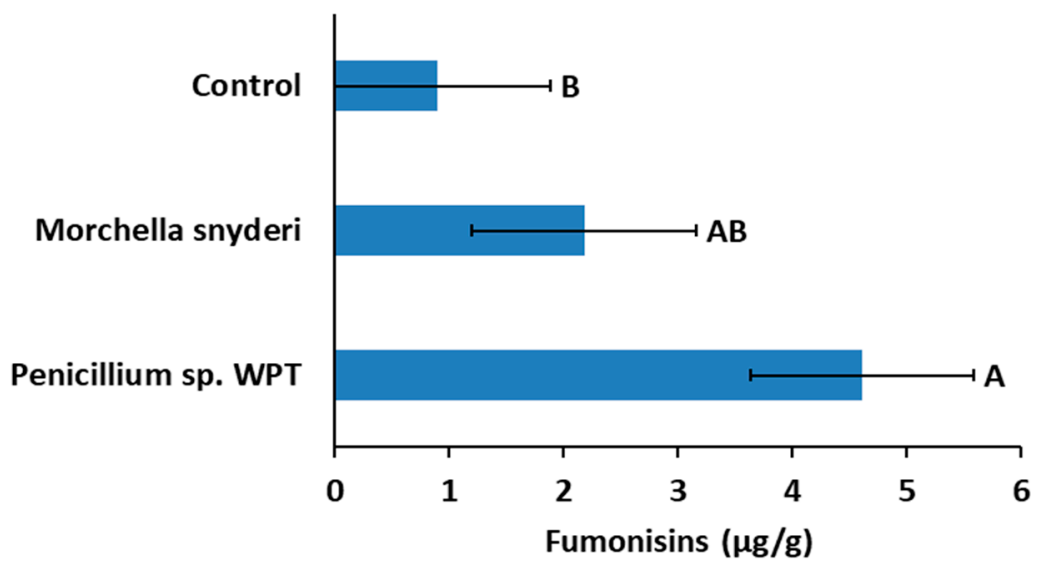

Figure 4. Applications of Penicillium WPT to seed at planting resulted in significant increases in fumonisin concentrations in mature seed. Application of Morchella snyderi slightly but not significantly increased fumonisin concentration in seed. Fumonisins can be systemically transported in plant solution. Error bars indicate standard error $(\alpha=0.05)$.

This ability to alter production of mycotoxins systemically was confirmed to be long-term in carry-over effects in the 2016 field trial. Mycotoxin concentrations in ears of plants grown from seeds from the 2015 silk inoculation trial were highly variable compared to concentrations in the 2015 seed (Figure 5), but effects of antagonists were observed to carry over to the second generation. Plants grown from seeds inoculated at silk with $M$. snyderi had $47 \%$ less DON $(p=0.0025)$ than plants grown from untreated seed (Figure 5b). 

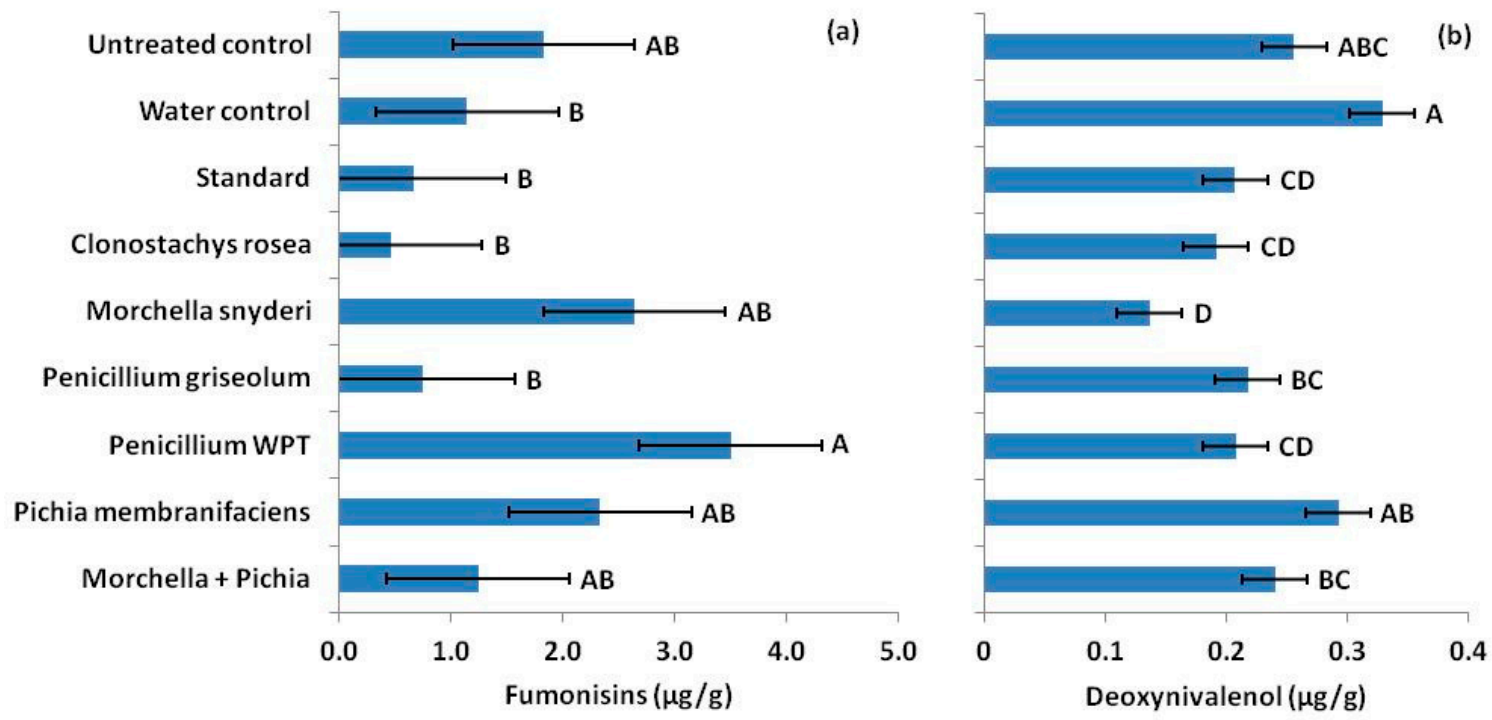

Figure 5. Antagonists applied emerging silks in an F1 hybrid increase in 2015 stimulated carry-over effects in the susceptible F1 hybrid the following year (2016). (a) Although average fumonisin content was both increased and reduced among treatments, differences were not significant. (b) However, seed from the Morchella snyderi treatment had half the DON concentrations of the untreated (dry) control. Error bars indicate standard error $(\alpha=0.05)$.

\subsection{Putative Antagonists and Fusarium Colonization of Seed In Situ}

Putative antagonists strongly altered mycotoxin production by Fusarium species locally, systemically, and across generations in the field, and effects on the colonization of mature kernels were also observed by qPCR. However, antagonism did not result in reductions in seedborne Fusarium. In fact, seed treated at silking with Penicillium WPT had over 6 times more total Fusarium DNA to seed DNA than untreated controls and 15 times more F. verticillioides (Figure 6). The abundance of F. verticillioides relative to total Fusarium was unaffected by Penicillium WPT. By contrast, C. rosea, P. griseolum and the combination treatment of Morchella and Pichia more than doubled the ratio of F. verticillioides to total Fusarium (Figure 7).

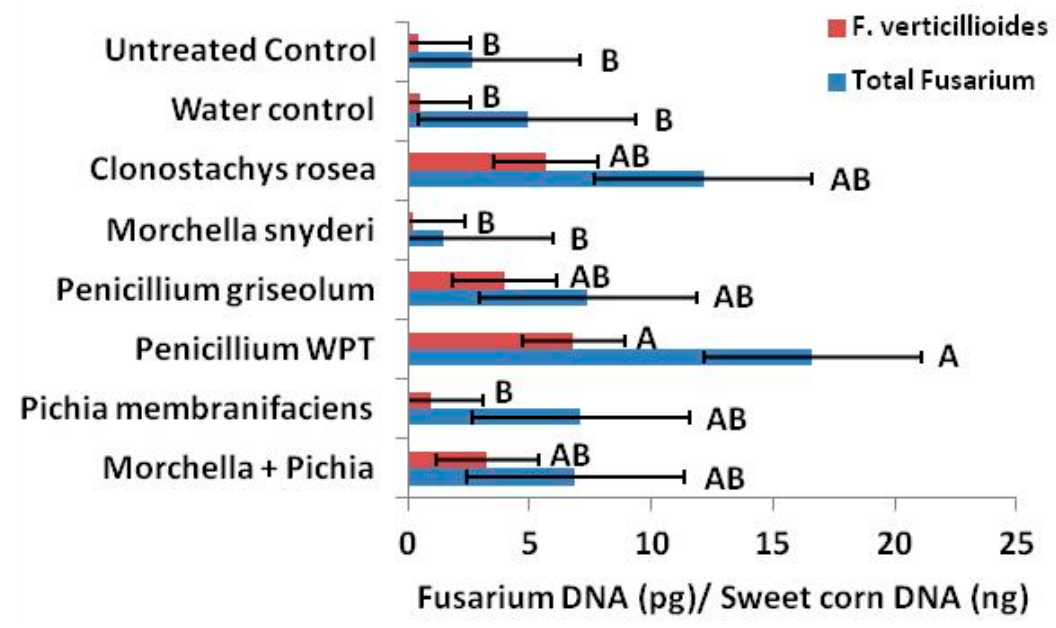

Figure 6. Quantitative PCR mostly showed increases in Fusarium colonization with the addition of fungal inocula applied to developing ears via emerging silks. Inoculation of silk with Penicillium WPT strongly increased both total Fusarium and F. verticillioides presence. Morchella snyderi was the only silk inoculation treatment that reduced colonization of both $F$. verticillioides and total Fusarium but not significantly. Error bars indicate standard error $(\alpha=0.05)$. Letters above red or blue bars indicate significant differences within a treatment set (F. verticillioides, red; Total Fusarium, blue). 


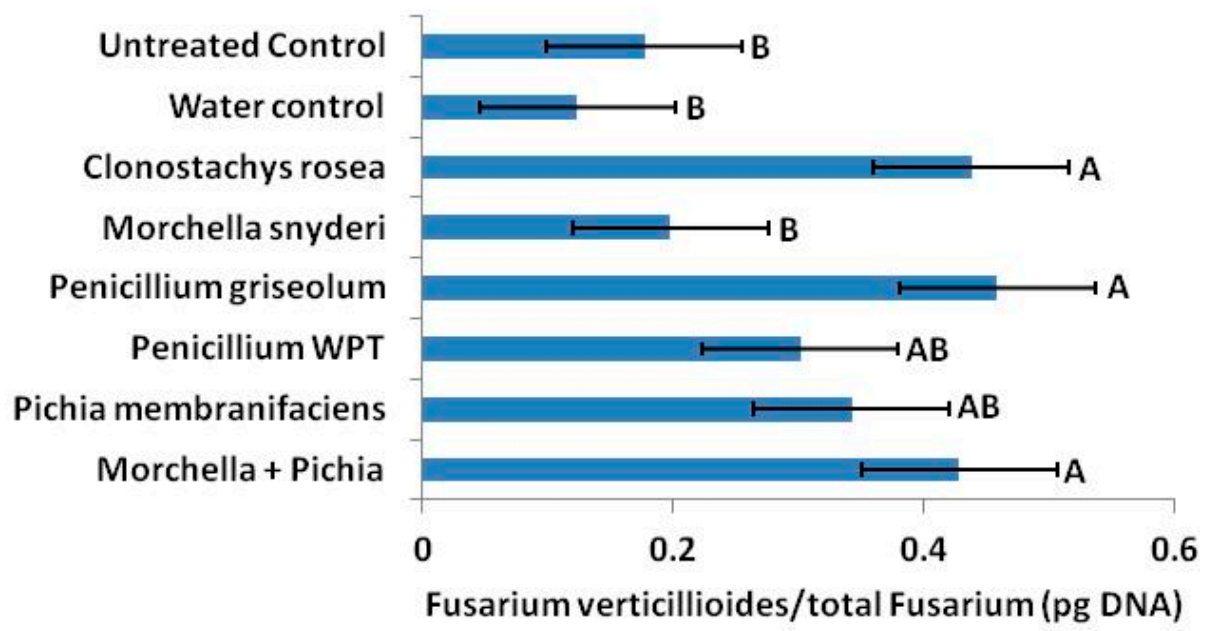

Figure 7. Although concentrations of Fusarium in mature seed were not significantly influenced, applications of C. rosea, P. griseolum, and Morchella + Pichia had a higher average ratio of $F$. verticillioides to total Fusarium than the untreated (dry) control $(p<0.05)$. Error bars indicate standard error $(\alpha=0.05)$.

\section{Discussion}

When challenged with fungal antagonists, Fusarium species from sweet corn seed fields in S. Idaho, USA, responded with detectable changes in mycotoxin production even while having no effect on the overall populations of the pathogens in seed. Not only were mycotoxin concentrations changed by direct antagonistic interactions in vitro but were altered by inoculations of antagonists locally in the ear. Moreover, inoculation with the novel Penicillium sp. WPT and Morchella snyderi effected systemic changes in accumulation of those fumonisins and DON in the seed.

Fumonisins [10] and DON [11] are known to move systemically in plants, concentrating in tissues without colonization of those tissues by their fungal producers. That single microbes applied either to the seed at planting (Figure 4) or to developing ears in silk (Figure 5) could alter those concentrations in first- and second-generation seed lots (respectively) in a complex abiotic and biotic environment would indicate that far more than the Fusarium species themselves are involved in mycotoxin accumulation in cereals. Whether these toxins are produced by Fusarium species as defense compounds cannot be determine by our study. However, challenging Fusarium species with antagonists did promote changes in both fumonisin and DON concentrations relative to unchallenged Fusarium both in vitro and in situ, indicating that other microbes in the ears of sweet corn or maize may strongly influence bioaccumulation of these toxins.

The novel species Penicillium sp. isolate WPT mostly stimulated fumonisin production by Fusarium both in vitro and in situ. The strongest effects of inoculation with the antagonist were systemic in the 2015 field trial in which seed was treated prior to planting. Mature seed produced on plants that developed from treated seed averaged five times the fumonisin content of seed from untreated control plants. It is interesting to note that Penicillium WPT produces griseofulvin (J. David Miller, Carlton U. ON CA, personal communication), an anti-fungal used pharmaceutically and known to suppress fungal disease in crops [49,50]. Griseofulvin has been shown to move systemically in plants [51,52], including major grass crops such as wheat, even from soil-borne microbes [53].

In vitro, antagonism by Penicillium WPT stimulated fumonisin production by F. verticillioides but reduced production by F. proliferatum. Penicillium griseolum had almost the opposite effects: it reduced fumonisin output by F. verticillioides while having no effect on $F$. proliferatum fumonisins. Penicillium griseolum had no effect either locally or systemically in situ, despite producing low concentrations of griseofulvin (J. David Miller, Carlton U. ON CA, personal communication), indicating that microbial biochemical interactions are likely specific to species. Griseofulvin accumulation was not determined either in vitro or in situ, so that no conclusions may be drawn as to its effects against Fusarium species 
from sweet corn and consequent effects on fumonisins. However, any biochemical antagonism between these Penicillia and Fusaria species would appear to be highly species dependent.

Just as antagonistic interactions were mostly species dependent, responses were also context dependent. Even the effects of Penicillium WPT antagonism on systemic fumonisin accumulation were weaker in the 2016 trials compared to 2015 trials. Whether the seed was inoculated at planting or the antagonist was introduced into the seed via the silks during ear development led to different systemic effects from M. snyderi on DON accumulation in seed. Treatment of seeds at planting with $M$. snyderi had no effect on DON in the subsequent crop. However, inoculation of developing ears at silking with $M$. snyderi reduced DON accumulation to half that of the untreated control in second-generation seed produced from that sown in 2016. By contrast, Fusarium responded to M. snyderi inoculation in developing ears by increasing DON concentrations in the seed. Different species within the genus have been known to produce anti-microbial compounds [54-56], but little is known about role of context in their efficacy. There is no record of anti-microbial activity for M. snyderi, but it both reduced conidial development by $F$. verticilioides and F. proliferatum in vitro and triggered reduced DON concentrations in field seed by systemic mechanisms.

Clonostachys rosea was isolated from immature kernels following silk inoculations, but we did not analyze either seed or PCR products to determine the persistence of fungal inoculants in samples tested for mycotoxins and Fusarium abundance. Although inoculations of Fusarium antagonists to seed and ears resulted in differing mycotoxin accumulations in subsequent seed, this response does not indicate a persistence of the inoculants within the ear microbiome. Effects could have been the result of more complicated interactions than direct antagonism between inoculants and target Fusarium species. Moreover, effects of antagonism might outlast persistence of the inoculant either form systemic biochemical accumulations (as might be possible with Penicillium WPT) or indirectly via changes within the existing microbial community of the developing kernel. Just as bioactive secondary metabolites like griseofulvin and the Fusarium mycotoxin zearalenone may persist in tissues lacking the physical presence of source microbes [51-53,57], changes in biochemical constituency and microbial communities (e.g., relative abundance) may result from initial inoculations and persist even while the inoculant.

The consequences of the complexity of the plant microbiome on seed mycotoxin concentrations cannot be underestimated. Fusarium proliferatum and F. verticillioides responded very differently to the same antagonists in controlled pairings. In situ, interactions with antagonists are no longer pairings but are subject to multiple species interactions. We isolated four Fusarium species from seed, immature kernels, and vegetative tissues from the field. Our findings were consistent with findings by Mohan and Wilson [58] with F. verticillioides being most common [48] followed by F. proliferatum. Fusarium temperatum and $F$. poae were isolated, but rare. However, none of our isolates produced DON in vitro, yet DON accumulated in mature seed and concentrations varied with antagonist interactions. It is likely that other Fusarium species are present in the system that were not successfully isolated. For example, F. graminearum has been repeated isolated from wheat heads in S. Idaho [59]. Fusarium species are known to antagonize each other $[35,38,39]$. Moreover, genetic variation among strains of the same Fusarium sp. vary in mycotoxin production [20]. From our own data we saw that interactions among Fusarium species (Figure 1) changed fumonisin concentrations. Although only one or two species may produce a particular mycotoxin in situ, other Fusarium present may influence accumulation and concentrations in seed.

In addition to multiple Fusarium species, Penicillium, Aspergillus, Rhizopus, Cladosporium and Trichoderma species are known to be present in sweet corn seed [44,60,61]. We obtained our isolate of Pichia membranifaciens as well as several unidentified bacteria and fungi from seed produced in 2014 field trials. In vitro, P. membranifaciens effectively suppressed both growth and fumonisin production by $F$. proliferatum and $F$. verticillioides. What effects these natural populations of antagonists might have as "third parties" to inocula can be partially seen in our 2016 field trials (Figure 5). Although M. snyderi alone reduced DON output to nearly half that in plants from untreated seed, concentrations in plants 
in the dual M. snyderi + P. membranifaciens inoculation treatment was similar to untreated. Thus, other microbes besides inocula play no small role in Fusarium output of mycotoxins.

Antagonistic inocula and perhaps naturally occurring antagonists are also likely to influence relative mycotoxin concentrations in seed by selectively antagonistic behavior toward Fusarium species. In our qPCR analysis, we determine that C. rosea, P. griseolum and the combination treatment $M$. snyderi + P. membranifaciens increased the ratio of F. verticillioides to total Fusarium (Figure 7).

Environmental effects, such as temperature and relative humidity, are known to contribute to variation in mycotoxin production $[21,38,62,63]$. To follow commercial rotation schedules, field sites changed each year of our study. We saw overall DON and fumonisin concentrations decline in 2016 compared to 2015, in the case of DON by an order of magnitude. This may have been in part due to changes in site location or the result of differences in precipitation and temperature patterns between the two years. Reductions in 2016 may also have resulted from shifts in species relative abundance of microbiota, specifically the Fusarium complex, within the ears.

Interactions in situ were mostly not predicted by in vitro results. Even where strong, antagonistic suppression of both fumonisin production and conidial development were observed in laboratory experiments, antagonists were mostly not suppressive in situ. For example, all antagonists strongly reduced conidial development of F. verticillioides in vitro; in situ, seed treated with Penicillium WPT at silking had over 15 times more F. verticillioides than untreated seed. Although in vitro interactions are frequently used to screen for microbial antagonism they often fail to have predictive value for field or in situ response variables [64-66].

If we would truly understand the variables that contribute to Fusarium mycotoxins in sweet corn seed or maize production, we must begin to study the system as a whole in situ, where effects of the biotic environment as well as the abiotic might be studied and dissected. If antagonistic inocula can have systemic effects on fumonisin and DON concentrations in sweet corn seed in the field, it is likely that we have underestimated the effects of the entire plant microbiome and microbial "biochemical warfare" [2] on mycotoxins.

\section{Materials and Methods}

\subsection{Isolating the Sweet Corn Fusarium Complex}

Fusarium species were isolated from seed samples collected from sweet corn field trials in Nampa ID, USA during 2014 and 2015 field trials. Seed samples were collected when kernels were immature and again at maturity. Seeds were plated onto $4 \%$ potato dextrose agar (PDA, Difco Laboratories, Inc., Detroit, MI, USA) and incubated for up to four weeks at $22{ }^{\circ} \mathrm{C}$. Fusarium isolates were aseptically transferred to pure culture on $4 \%$ PDA.

\subsection{Fusarium Species Identification}

Twelve isolates of Fusarium species were selected for sequence identification to represent individual morphotypes from the pure culture collection. Genomic DNA from the 12 cultured Fusarium isolates was amplified using the ITS-1F and LR3 and the EF1 and EF2 primer pairs. PCR products were checked by agarose gel electrophoresis and purified by $\mathrm{PEG} / \mathrm{NaCl}$ precipitation. These templates were sequenced with BIgDye3.1 (Applied Biosystems $₫$, Foster, CA, USA) using the same primers used for amplification and analyzed on an ABI3130XL Genetic Analyzer (Life Technologies, Inc., Thermo Fisher Scientific, Waltham, MA, USA) with an $80 \mathrm{~cm}$ capillary array. Forward and reverse read consensus sequences were checked against GenBank and candidate Fusarium species were identified from the EF1/2 sequences at $99-100 \%$ sequence identity and cross-validated by micromorphology.

\subsection{Selecting Fungal Antagonists}

Fungal antagonists of Fusarium species were selected for laboratory and field research using in vitro antagonism assays. Fungi of interest were obtained from indigenous plant microbiomes of the 
intermountain west, including Pseudotsuga menziesii var. glauca, Abies grandis, and Pinus ponderosa [67]. These fungi were previously observed to antagonize F. proliferatum isolated from P. menziesii in culture. A well-known mycoparasitic fungus Clonostachys rosea, isolated from roots of P. menziesii var. glauca was deliberately selected for its mycoparasistic activity, and a fungistatic yeast, Pichia membranifaciens isolated from sweet corn was also selected. New in vitro assays were designed to screen for antagonistic behavior by inoculating petri plates of $4 \%$ PDA with paired antagonists. A center line was drawn across the diameter of the plate. Fusarium species were inoculated into the center of the space on one side of the plate approximately $1 \mathrm{~cm}$ from the line. Directly opposite, one antagonist was inoculated onto the plate $1 \mathrm{~cm}$ from the line. Control plates were grown with only the Fusarium or an antagonist. Observations were taken of the plates for five to seven days at $22{ }^{\circ} \mathrm{C}$. Fungi that appeared to reduce growth and spread of Fusarium species across the center line of the plate and/or appeared to reduce growth or stimulate pigmented secondary metabolites with physical contact were subjected to a quantitative test in broth antagonism cultures.

\subsection{In Vitro Quantitative Tests of Antagonism}

Fusarium species were co-inoculated with selected antagonists in paired antagonism broth cultures with a Fusarium culture broth containing a solution of $2 \%$ sucrose, $2 \%$ glucose, $1.5 \%$ peptone, and $1 \%$ potato dextrose adjusted to a $\mathrm{pH}$ of 6.2 . Each $50-\mathrm{mL}$ culture contained either F. verticillioides or F. proliferatum and a single antagonist. Five antagonists observed to antagonize Fusarium species in vitro were tested: Clonostachys rosea, Morchella snyderi, Penicillium sp. nov. WPT, Penicillium griseolum, and P. membranifaciens. Control cultures contained only Fusarium species

To inoculate the broth cultures, two-week-old PDA plate cultures of each fungus (antagonists or Fusarium spp.) were flooded with sterile distilled water (SDW) and scraped with a bent, glass rod to loosen fungal propagules into solution. Propagules of $M$. snyderi, which forms heavy hyphal filaments and sclerotia on PDA, were loosened using a scalpel blade. One hundred microliters of Fusarium propagule solution were inoculated into each $100 \mathrm{~mL}$ test tube containing $50 \mathrm{~mL}$ of the broth solution for each antagonism. For each antagonism treatment, $100 \mu \mathrm{L}$ of antagonist propagule solution were added. Four replicate cultures were made for each treatment with four antagonist-free Fusarium cultures for a control. Fusarium proliferatum and F. verticillioides solutions were inoculated into their respective broth cultures at $10^{7}$ propagules per $\mathrm{ml}$. Starting cultures contained $2 \times 10^{5}$ propagules per $\mathrm{ml}$ of each pathogen. Inocula were not standardized among antagonists since effects among antagonists were not comparable due to highly different mechanisms of antagonism and the antagonists were not being compared. Starting concentrations in propagules per $\mathrm{ml}$ of broth were as follows: C. rosea, $7 \times 10^{4}$; M. snyderi, $7 \times 10^{1} ;$ P. griseolum, $3.8 \times 10^{4}$; Penicillium WPT, $3.4 \times 10^{5} ;$ P. membranifaciens, $1.2 \times 10^{4}$.

Following inoculation, the broth cultures were incubated for three days at $22{ }^{\circ} \mathrm{C}$ on a shaker at a constant $150 \mathrm{rpm}$. On day three, counts of Fusarium conidia were made in each culture using a Neubauer cell counting chamber. Counts were computed to the concentration of conidia per $\mathrm{ml}$.

\subsection{Mycotoxin ELISA-Direct Antagonisms}

Sequenced Fusarium isolates from the sweet corn seed isolate collection were assayed for their ability to produce mycotoxins. Three putative fumonisin producers and a single putative deoxynivalenol (DON) producer were assayed. Pure cultures of the four isolates were cultured on $4 \%$ PDA for ten days at $22{ }^{\circ} \mathrm{C}$. Ten grams of culture material (mycelium and agar) were removed from the plates and placed in jars. Fumonisins were extracted with 70\% HPLC-grade methanol at a ratio of 1:5 (10 g culture material: $50 \mathrm{~mL}$ methanol). DON extracts were made with deionized (DI) water at a 1:5 ratio (culture material: DI water). Extracts were then analyzed using ELISA 96-well mycotoxin enzyme kits (Agriquant ${ }^{\circledR}$, Romer Labs Inc., Newark, DE, USA) according to manufacturer's instructions. ELISA plates were scanned using a microplate spectrophotometer (Powerwave ${ }^{\mathrm{TM}}$, Bio-Tek Instruments, Winooski, VT, USA) to obtain optical density (OD) readings. Data were transformed using a standard curve to obtain concentrations $(\mu \mathrm{g} / \mathrm{g})$. 
Effects of antagonisms on mycotoxin production were determined with a series of in vitro assays pairing Fusarium species with antagonists. Five antagonists were paired with two fumonisin producers: C. rosea, M. snyderi, Penicillium sp. nov. WPT, P. griseolum, and P. membranifaciens. The two fumonisin-producing Fusarium species were also paired with the other two sequenced Fusarium species to determine potential mycotoxin interactions among the isolates. Fumonisin producers were inoculated onto $4 \%$ PDA plates $1 \mathrm{~cm}$ from the center of the plates. On the opposing side of each plate, also $1 \mathrm{~cm}$ from the center of the plate, one of the antagonists or co-occurring Fusarium isolates was plated. Eight plates were made for each of the pairings with co-occurring Fusarium isolates or fungal antagonists, respectively. The positive control plates consisted of the fumonisin producers without competition in the plates. Negative controls were generated for each of the five antagonists by plating each antagonist alone. Only four plates were made for each negative control. After three weeks, a 2 -cm wide by $9 \mathrm{~cm}$ long strip of agar and fungal material running perpendicular to the dual cultures was removed from each plate. Fumonisins were extracted by submerging each strip in $70 \%$ methanol at a ratio of 1:5 (w/v, culture material:methanol). Extracts were analyzed using the ELISA technique as previously described.

\subsection{Testing Effects of Antagonism in Situ}

\subsubsection{Field Trial-Silk Inoculations}

The summer 2015 two in situ antagonism studies were sown in Nampa ID, USA. The first antagonism study examined effects of direct antagonisms via inoculation of emerging silks of a susceptible inbred line (L2) on mycotoxin accumulation in and Fusarium colonization of developing seed. The study also functioned as a seed increase to generate antagonist-carrying F1 hybrid seed to be sown the following year (2016) to study potential carry-over and systemic effects from seed transmission of the inoculants to an F2 generation. The female inbred was sown in early June 2015 in a standard F1 hybrid increase design with four female rows bracketed by two male rows. The male line was sown approximately two weeks previous to planting the female line.

In late July (23 July 2015), emerging silks were inoculated with eight different antagonism treatments: C. rosea, M. snyderi, P. griseolum, Penicillium WPT, P. membranifaciens, $M$. snyderi $+P$. membranifaciens, a sterile water control, and an untreated control that received no wet applications. Fungal propagules (spores and macerated hyphae) were suspended in sterile distilled water at concentrations of $10^{4}$ propagules per $\mathrm{ml}$ for $\mathrm{M}$. snyderi and $10^{7}$ propagules per $\mathrm{ml}$ for all other inocula. Inocula were not standardized among antagonists since effects among antagonists were not comparable due to highly different mechanisms of antagonism. Antagonists were applied to emerging silks using hand-held atomizers calibrated to $5 \mathrm{~mL}$ of suspension per ear. The M. snyderi $+P$. membranifaciens treatment received $2.5 \mathrm{~mL}$ of each antagonist for a total of $5 \mathrm{~mL}$ of inocula. This inoculation was repeated for three nights with inoculations beginning at approximately 6:30PM MST so that each treatment received $15 \mathrm{~mL}$ of suspension. Temperature and relative humidity at the silking zone were monitored over a $36-\mathrm{h}$ period beginning the first evening. Treatments were arranged in an 8-block randomized block design at the head of the increase field with 10 plants treated per treatment per block. A total of 640 plants were used in the experiment.

Mature ears were harvested September 17. Ears were dried at $34^{\circ} \mathrm{C}$. Subsamples for each ear were randomly selected for quantitative PCR testing and ELISA mycotoxin assays. Later, seed from individual ears was bulked by treatment for a 2016 planting.

\subsubsection{Field Trial—Seed Treatment for Systemic Effects}

The second trial to investigate possible systemic antagonism was planted in May 2015. Antagonist-treated seeds from the Fusarium-susceptible inbred L2 were sown in a replicated trial in Nampa ID. Treatments were blocked in replicated ranges of 12 rows. Each treatment was separated by a buffer row, the seeds of which were treated with standard pesticide treatments. Three antagonist 
treatments were tested: M. snyderi, Penicillium WTP, and a control. The antagonists were propagated on food-grade yellow cornmeal. The purpose of the cornmeal was to serve as both a culture substrate and an inoculum carrier. Inoculated cornmeal was crushed, mixed, and moistened with deionized (DI) water. The inocula were applied to seeds prior to sowing with the seeds and cornmeal carrier mixed thoroughly to coat each seed. Plain moistened cornmeal was used for the control treatment.

Starting September 25, ears were harvested on a per row basis with a 15-foot row length as a replicate. Ears were dried at $34^{\circ} \mathrm{C}$, and subsamples of mature seeds from each row were taken for mycotoxin ELISA assays.

\subsubsection{Field Trial-Carry-Over Effects}

The F1 hybrid seeds generated from the 2015 silk inoculation trial were sown for a final field trial in May 2016. Following 2015 analyses, remaining seed was bulked by treatment. Random samples of the bulked seed were taken for sowing in the 2016 field trail. Six-row seed ranges were sown with a single treatment in a randomized block design with ranges for each treatment represented in each of four blocks. There were nine treatments sown with a total of 36 ranges. The treatments included seeds from all eight silk treatments from 2015: untreated control, water control, C. rosea, M. snyderi, P. griseolum, Penicillium WPT, P. membranifaciens, and seeds from the M. snyderi + P. membranifaciens inoculation. The seeds generated from the 2015 trial were hybrid seeds. They received no further treatment in 2016 prior to sowing. The ninth treatment was a subsample of the 2016 untreated control to which was applied the standard fungicide/insecticide seed treatment prior to sowing.

Ears were harvested by the 10 -foot row (4 rows per range) in early October. Ears were dried in a commercial drier at a standard $34^{\circ} \mathrm{C}$. Seed subsamples of this F2 generation were taken from each row for ELISA analyses of mycotoxin content.

\subsubsection{Mycotoxin ELISA-2015 and 2016 Field Seed}

Seeds from the 2015 field trials and the 2016 field trial were analyzed for the presence and content of both fumonisins and DON. For mycotoxin analyses of the 2015 seedborne antagonism trial, samples were bulked by row and three randomly selected rows were analyzed per treatment. Samples from the 2015 silk-inoculation field trial and the 2016 field trial were bulked by row and eight random rows from each antagonism treatment were selected for analysis. Three randomly selected samples were analyzed for both DON and fumonisins from the 2015 seed treatment field trial. Samples were ground with a coffee mill, and the mill was sanitized between samples. Fumonisins were extracted from ground samples with 70\% HPLC grade methanol at a ratio of 1:5 (w/v, sample:methanol). DON was extracted with DI water at a ratio of 1:5 (w/v, sample:DI water). Mycotoxin concentrations of extracts were determine using ELISA 96-well mycotoxin enzyme kits (Agriquant ${ }^{\circledR}$, Romer Labs Inc., Newark, DE, USA) according to manufacturer's instructions. ELISA plates were scanned using a microplate spectrophotometer (Powerwave ${ }^{\mathrm{TM}}$, Bio-Tek Instruments, Winooski, VT, USA) to obtain OD readings. Data were transformed using a standard curve to obtain concentrations $(\mu \mathrm{g} / \mathrm{g})$.

\subsection{Molecular Determination of Fusarium Antagonism}

Finally, we used the quantitative polymerase chain reaction (qPCR) technique to compare quantities of Fusarium DNA relative to seed yield (plant DNA) from a subsample of mature seeds from the 2015 silk inoculation field trial. Only 2015 seed was analyzed due to resource constraints. Sixteen randomly selected ears were taken from each of the eight treatments, and four ears were then pooled to generate four replicates per antagonist for analysis. Thirty-gram samples of dry seeds (average 240 kernels) from each treatment and control plot were lyophilized $\mathrm{O} / \mathrm{N}$ and then ground using a coffee/spice grinder (Kitchen Aid model BCG11108) which has a removable stainless-steel bowl to allow convenient cleaning between samples. Sub-samples of $100 \mathrm{mg}$ of the ground seeds were ground further to a powder on a MixerMill 300 with a tungsten carbide bead in a $2 \mathrm{ml}$ Collection Tube (Qiagen N.V., Venlo, Limburg, Netherlands). 


\subsubsection{Genomic DNA Extraction}

DNA was extracted from the powdered seed biomass using the CTAB method [68]. The CTAB DNA preps were then subjected the PEG/NaCl precipitation. These DNAs were characterized by UV optical absorbance using a microplate spectrophotometer (Epoch ${ }^{\mathrm{TM}}$, BioTek Instruments, Inc., Winooski, VT, USA) and by successful endpoint PCR using tEF1 and ITS fungal primers. For greater consistency, we followed the CTAB extraction with further processing using a Qiagen DNeasy Plant kit according to the manufacturer's instructions (Qiagen N.V., Venlo, Limburg, Netherlands) to remove residual PCR inhibitors present in the seeds. DNA quality was checked by UV optical absorbance and concentrations were measured using a Qubit ${ }^{\circledR}$ fluorimeter (Invitrogen ${ }^{\mathrm{TM}}$, Thermo Fisher Scientific, Waltham, MA, USA) with a QuantIt ${ }^{\mathrm{TM}}$ broad range kit (Invitrogen ${ }^{\mathrm{TM}}$, Thermo Fisher Scientific, Waltham, MA, USA).

Genomic DNA was also extracted from the sequenced Fusarium strains isolated from plants in the field trials conducted during 2014 and 2015. Fusarium strains were cultured on 4\% PDA plates. Fungal biomass was scraped off the agar surface and DNA was extracted using the Qiagen DNeasy Plant 96 kit according to the manufacturer's instructions (Qiagen N.V., Venlo, Limburg, Netherlands). DNA quality was checked by UV optical absorbance using an Epoch microplate spectrophotometer (BioTek Instruments, Inc., Winooski, VT, USA). Concentrations were measured using a Qubit ${ }^{\circledR}$ fluorimeter and a QuantIt ${ }^{\mathrm{TM}}$ broad range assay kit (Invitrogen ${ }^{\mathrm{TM}}$, Thermofisher Scientific, Waltham, MA, USA).

\subsubsection{Fusarium Genus Primer Design}

Two measures of Fusarium content were quantified in the seeds: total Fusarium content (genus level DNA) and the most common species, F. verticillioides, isolated from the Nampa ID trials. Q-PCR for F. verticillioides was handled as a separate analysis. Nicolaisen et al. [69] developed Fusarium species-specific primer pairs from within the $\mathrm{tEF} 1 \alpha$ gene for 11 Fusarium species which are commonly found in cereals and other crops. During our study four species of Fusarium were repeatedly isolated from the seeds and identified. Three of these had specific qPCR primers developed by Nicolaisen et al. [69]; the fourth had not.

To identify regions of sequence conservation in translation elongation factor alpha gene among all Fusarium species of interest we aligned all the tEF1 $\alpha$ sequences available in GenBank for 13 Fusarium species that are commonly found in crops in temperate regions. The species included in the alignment were F. avenaceum, F. culmorum, F. equiseti, F. graminearum, F. langsethiae, F. poae, F. proliferatum, F. pseudograminearum, F. sporotrichioides, F. subglutinans, F. temperatum, F. tricinctum, and F. verticillioides. The alignment of 504 sequences was done in CLCBio Main Workbench software (Qiagen Bioinformatics, Aarhus, Denmark). We were able to identify a region where an amplicon of between 50 and 100 bases could be produced for about $90 \%$ of the aligned sequences using an individual forward primer sequence (FsGns547F ACGCCTGGGTTCTTGACA) and a mixture of 3 similar reverse primer sequences (Rev1 FsGnsallR CGGTGACATAGTAGCGAGGAGT, Rev2 FsGnspoaeR GGTGACATAGTAGCGGGGAGT, and Rev3 FsGnstmprR TGACAGTGACATAGTAGCGAGGAG). The forward primer was used at a final concentration of $0.167 \mu \mathrm{M}$ in the qPCR reaction and the reverse primers were used at $0.133 \mu \mathrm{M}$ each. All primers have a predicted melting temperature (Tm) of roughly $62{ }^{\circ} \mathrm{C}$.

\subsubsection{Quantitative PCR Assay}

The amplification conditions and primer concentrations were tested in endpoint PCRs and analyzed on 5\% high-resolution blend agarose gels (Lonza, Basel, Switzerland). Endpoint PCRs were done using MyTaq Red 2x master mix (Bioline, London, UK). Primer dimers which were formed at typical endpoint PCR primer concentrations were avoided by dilution of the primers. Only the expected products were made when Fusarium DNA was used as template and the selected primer concentrations were used. Some higher molecular weight material was sometimes produced especially when antagonist-treated seed DNA was used as template. We largely avoided this by keeping the extension times to a minimum and using a higher annealing temperature in the first 5 cycles, in a 2-step 
cycling program. All qPCRs were performed using SYBR Fast $2 \times$ Mix reagent (Bioline, London, UK) and reactions were carried out on an MJ Research Chromo4 (BioRad, Des Plaines, IL, USA) instrument. Once the cycling conditions were optimized for qPCR clean melting curves were obtained and standard curves were linear with from $10 \mathrm{ng}$ down to $1 \mathrm{pg}$ of Fusarium DNA from the four Fusarium species isolated during this study. The identities of the PCR products were checked by Sanger sequencing [70] and found to be as expected.

The thermal cycling program for the total Fusarium genus qPCR assay was as follows:
1. $95^{\circ} \mathrm{C}$
$3 \mathrm{~min}$
2. $95^{\circ} \mathrm{C}$
$5 \mathrm{~s}$
3. $68^{\circ} \mathrm{C}$
$8 \mathrm{~s}$
4. Go to 2
$2 \times$
5. $95^{\circ} \mathrm{C}$
$5 \mathrm{~s}$
6. $67^{\circ} \mathrm{C}$
$8 \mathrm{~s}$
7. Go to 5
$1 \times$
8. $95^{\circ} \mathrm{C} \quad 5 \mathrm{~s}$
9. $66^{\circ} \mathrm{C} \quad 18 \mathrm{~s}$
10. Plate read
11. Go to $8 \quad 34 \times$
12. Melting curve $70^{\circ} \mathrm{C}$ to $95^{\circ} \mathrm{C}$ in $0.5^{\circ} \mathrm{C}$ increments, $5 \mathrm{~s}$ per step.

The cycling program for the $F$. verticillioides primers was the same but with a $2{ }^{\circ} \mathrm{C}$ lower annealing/extension temperature.

The quantity of plant DNA in each seed DNA sample was measured by qPCR using the hor1F and hor2R plant-specific primers as described in Nicolaisen et al. [69]. Seed DNA from one test plot sample that gave the same DNA concentrations in both the Qubit ${ }^{\circledR}$ and Epoch ${ }^{\mathrm{TM}}$ DNA assays was used to construct the standard curve for the plant DNA assay. As determined by Qubit ${ }^{\circledR}$ measurement, $25 \mathrm{ng}$ of seed DNA was used in each qPCR for Fusarium assays.

Spiking excess pure fungal DNA into qPCRs containing seed DNA showed that there was some PCR inhibition associated with the seed DNA extracts. A correction factor was calculated by spiking two samples of seed DNA from each of the 8 sets of test plot samples with an excess of $F$. verticillioides DNA and comparing the calculated quantity to that obtained with the fungal DNA alone. This was done for both the Fusarium genus assay and the F. verticillioides assay.

\subsection{Statistical Analyses}

Propagule counts for Fusarium species in laboratory antagonism assays were non-parametric and were analyzed using the Kruskal-Wallis Wilcoxon rank score estimate in the NPAR1WAY procedure using SAS/STAT ${ }^{\circledR}$ software (SAS ${ }^{\circledR}$ 9.4, 2017, SAS Institute Inc., Cary, NC, USA) [71]. Mean comparisons were made using standard error as computed from mean score standard deviations.

All absorbance data from mycotoxin ELISA assays were transformed to concentrations using a logit-log function (Romer Labs, Inc. Newark, DE, USA) to generate a standard curve. Concentration data were then analyzed by analysis of variance and Fisher's LSD and least squares mean comparisons using the GLM and MIXED procedures in SAS.

Results of the Fusarium genus qPCRs and F. verticillioides qPCRs, each measured on triplicate samples, were corrected for inhibition in each set of test plot samples and expressed as pg of total Fusarium or F. verticillioides DNA per ng of plant DNA. qPCR data analysis was performed using the web-based PCR Miner software package [72]. Threshold cycle values were standardized for conversion to picograms of Fusarium DNA per nanograms of seed DNA. These data were then analyzed using the Kruskal-Wallis Wilcoxon rank score estimate in the NPAR1WAY procedure in SAS. Mean comparisons were made using standard error as computed from mean score standard deviations.

Author Contributions: Conceptualization, G.N. and M.E.R.; Methodology, M.E.R. and B.G.; Validation, M.E.R. and B.G.; Formal Analysis, M.E.R. and B.G.; Investigation, M.E.R. and B.G.; Resources, G.N. and B.G.; Data Curation, M.E.R. and B.G.; Writing-Original Draft Preparation, M.E.R.; Writing-Review \& Editing, B.G. and 
G.N.; Visualization, M.E.R.; Supervision, G.N.; Project Administration, M.E.R.; Funding Acquisition, G.N., M.E.R. and B.G.

Funding: This study was funded by HM-Clause Vegetable Seeds, Inc.

Acknowledgments: The authors would like to thank Scott Gold of USDA ARS, Athens, GA and Nicole Crenshaw of University of Georgia, Athens, GA for sequencing our P. membranifaciens isolate. Publication of this article was funded by the University of Idaho Open Access Publishing Fund.

Conflicts of Interest: The authors declare no conflict of interest. Field researchers with HM-Clause assisted with planting of field research plots and data collection.

\section{References}

1. Mlot, C. Antibiotics in nature: Beyond biological warfare. Science 2009, 324, 1637-1639. [CrossRef] [PubMed]

2. Czárán, T.L.; Hoekstra, R.F.; Pagie, L. Chemical warfare between microbes promotes biodiversity. Proc. Natl. Acad. Sci. USA 2002, 99, 786-790. [CrossRef] [PubMed]

3. Janzen, D.H. Why fruits rot, seeds mold, and meat spoils. Am. Nat. 1977, 111, 691-713. [CrossRef]

4. Bérdy, J. Bioactive microbial metabolites. J. Antibiot. 2005, 58, 1-26. [CrossRef] [PubMed]

5. Placinta, C.M.; D'mello, J.P.F.; Macdonald, A.M.C. A review of worldwide contamination of cereal grains and animal feed with Fusarium mycotoxins. Anim. Feed Sci. Technol. 1999, 78, 21-37. [CrossRef]

6. Miedaner, T.; Bolduan, C.; Melchinger, A.E. Aggressiveness and mycotoxin production of eight isolates each of Fusarium graminearum and Fusarium verticillioides for ear rot on susceptible and resistant early maize inbred lines. Eur. J. Plant Pathol. 2010, 127, 113-123. [CrossRef]

7. Stenglein, S.A. Fusarium poae: A pathogen that needs more attention. J. Plant Pathol. 2009, 91, $25-36$.

8. National Grain and Feed Association. FDA Mycotoxin Regulatory Guidance: A Guide for Grain Elevators, Feed Manufacturers, Grain Processors and Exporters. 2011. Available online: https://www.ngfa.org/wpcontent/uploads/NGFAComplianceGuide-FDARegulatoryGuidanceforMycotoxins8-2011.pdf (accessed on 24 May 2017).

9. United States Food and Drug Administration. Mycotoxin Handbook. 2015. Available online: https: //www.gipsa.usda.gov/fgis/handbook/MycotoxinHB/MycotoxinHandbook_2016-07-12.pdf (accessed on 24 May 2017).

10. Baldwin, T.T.; Zitomer, N.C.; Mitchell, T.R.; Zimeri, A.M.; Bacon, C.W.; Riley, R.T.; Glenn, A.E. Maize seedling blight induced by Fusarium verticillioides: Accumulation of Fumonisin B1 in leaves without colonization of the leaves. J. Agric. Food Chem. 2014, 62, 2118-2125. [CrossRef]

11. Covarelli, L.; Beccari, G.; Steed, A.; Nicholson, P. Colonization of soft wheat following infection of the stem base by Fusarium culmorum and translocation of deoxynivalenol to the head. Plant Pathth. 2012, 61, 1121-1129. [CrossRef]

12. Desjardins, A.E.; Proctor, R.H.; Bai, G.; McCormick, S.; Shaner, G.E.; Buechley, G.; Hohn, T.M. Reduced virulence of trichothecene-nonproducing mutants of Gibberella zeae in wheat field tests. Mol. Plant Microbe Interact. 1996, 9, 775-781. [CrossRef]

13. Harris, L.J.; Desjardins, A.E.; Plattner, R.D.; Nicholson, P.; Butler, G.; Young, J.C.; Weston, G.; Proctor, R.H.; Hohn, T.M. Possible role of trichothecene mycotoxins in virulence of Fusarium graminearum on maize. Plant Dis. 1999, 83, 954-960. [CrossRef] [PubMed]

14. Quesada-Ocampo, L.M.; Al-Haddad, J.; Scruggs, A.C.; Buell, C.R.; Trail, F. Susceptibility of maize to stalk rot caused by Fusarium graminearum deoxynivalenol and zearalenone mutants. Phytopathology 2016, 106, 920-927. [CrossRef] [PubMed]

15. Gu, Q.; Tahir, H.A.S.; Zhang, H.; Huang, H.; Ji, T.; Sun, X.; Wu, L.; Wu, H.; Gao, X. Involvement of FvSet1 in fumonisin b1 biosynthesis, vegetative growth, fungal virulence, and environmental stress responses in Fusarium verticillioides. Toxins 2017, 9, 43. [CrossRef] [PubMed]

16. Ridenour, J.B.; Bluhm, B.H. The novel fungal-specific gene FUG1 has a role in pathogenicity and fumonisin biosynthesis in Fusarium verticillioides. Mol. Plant Pathol. 2017, 18, 513-528. [CrossRef]

17. Ridenour, J.B.; Smith, J.E.; Bluhm, B.H. The HAP complex governs fumonisin biosynthesis and maize kernel pathogenesis in Fusarium verticillioides. J. Food Prot. 2016, 79, 1498-1507. [CrossRef] 
18. Desmond, O.J.; Manners, J.M.; Stephens, A.E.; Maclean, D.J.; Schenk, P.M.; Gardiner, D.M.; Munn, A.L.; Kazan, K. The Fusarium mycotoxin deoxynivalenol elicits hydrogen peroxide production, programmed cell death and defense responses in wheat. Mol. Plant Pathol. 2008, 9, 435-445. [CrossRef]

19. Leslie, J.F.; Plattner, R.D.; Desjardins, A.E.; Klittich, C.J. Fumonisin B1 production by strains from different mating populations of Gibberella fujikuroi (Fusarium section Liseola). Phytopathology 1992, 82, 341-345. [CrossRef]

20. Proctor, R.H.; Plattner, R.D.; Desjardins, A.E.; Busman, M.; Butchko, R.A. Fumonisin production in the maize pathogen Fusarium verticillioides: Genetic basis of naturally occurring chemical variation. J. Agric. Food Chem. 2006, 54, 2424-2430. [CrossRef]

21. Munkvold, G.P. Epidemiology of Fusarium diseases and their mycotoxins in maize ears. In Epidemiology of Mycotoxin Producing Fungi; Xu, X., Bailey, J.A., Cooke, M., Eds.; Springer: New York, NY, USA, 2003; pp. 705-713.

22. Estrada, A.E.R.; Jonkers, W.; Kistler, H.C.; May, G. Interactions between Fusarium verticillioides, Ustilago maydis, and Zea mays: An endophyte, a pathogen, and their shared plant host. Fungal Genet. Biol. 2012, 49, 578-587. [CrossRef]

23. Lee, K.; Pan, J.J.; May, G. Endophytic Fusarium verticillioides reduces disease severity caused by Ustilago maydis on maize. FEMS Microbiol. Lett. 2009, 299, 31-37. [CrossRef]

24. Pal, K.K.; Tilak, K.V.B.R.; Saxcna, A.K.; Dey, R.; Singh, C.S. Suppression of maize root diseases caused by Macrophomina phaseolina, Fusarium moniliforme and Fusarium graminearum by plant growth promoting rhizobacteria. Microbiol. Res. 2001, 156, 209-223. [CrossRef] [PubMed]

25. Bacon, C.W.; Yates, I.E.; Hinton, D.M.; Meredith, F. Biological control of Fusarium moniliforme in maize. Environ. Health Perspect. 2001, 109 (Suppl. 2), 325-332.

26. Bacon, C.W.; Hinton, D.M.; Porter, J.K.; Glenn, A.E.; Kuldau, G. Fusaric acid, a Fusarium verticillioides metabolite, antagonistic to the endophytic biocontrol bacterium Bacillus mojavensis. Can. J. Bot. 2004, 82, 878-885. [CrossRef]

27. Mousa, W.K.; Shearer, C.R.; Limay-Rios, V.; Zhou, T.; Raizada, M.N. Bacterial endophytes from wild maize suppress Fusarium graminearum in modern maize and inhibit mycotoxin accumulation. Front. Plant Sci. 2015, 6, 805. [CrossRef] [PubMed]

28. Pereira, P.; Nesci, A.; Castillo, C.; Etcheverry, M. Impact of bacterial biological control agents on fumonisin B 1 content and Fusarium verticillioides infection of field-grown maize. Biol. Control 2010, 53, 258-266. [CrossRef]

29. Velluti, A.; Marín, S.; Gonzalez, R.J.; Ramos, A.; Sanchis, V. Fumonisin B1, zearalenone and deoxynivalenol production by Fusarium moniliforme, F proliferatum and $F$ graminearum in mixed cultures on irradiated maize kernels. J. Sci. Food Agric. 2001, 81, 88-94. [CrossRef]

30. Keyser, Z.; Vismer, H.F.; Klaasen, J.A.; Snijman, P.W.; Marasas, W.F.O. The antifungal effect of fumonisin B 1 on Fusarium and other fungal species. S. Afr. J. Sci. 1999, 95, 455-457.

31. Bacon, C.W.; Hinton, D.M.; Hinton, A. Growth-inhibiting effects of concentrations of fusaric acid on the growth of Bacillus mojavensis and other biocontrol Bacillus species. J. Appl. Microbiol. 2006, 100, 185-194. [CrossRef] [PubMed]

32. Yates, I.E.; Meredith, F.; Smart, W.; Bacon, C.W.; Jaworski, A.J. Trichoderma viride suppresses fumonisin B1 production by Fusarium moniliforme. J. Food Prot. 1999, 62, 1326-1332. [CrossRef] [PubMed]

33. Chatterjee, S.; Kuang, Y.; Splivallo, R.; Chatterjee, P.; Karlovsky, P. Interactions among filamentous fungi Aspergillus niger, Fusarium verticillioides and Clonostachys rosea: Fungal biomass, diversity of secreted metabolites and fumonisin production. BMC Microbiol. 2016, 16, 83. [CrossRef]

34. Hebbar, K.P.; Davey, A.G.; Dart, P.J. Rhizobacteria of maize antagonistic to Fusarium moniliforme, a soil-borne fungal pathogen: Isolation and identification. Soil Biol. Biochem. 1992, 24, 979-987. [CrossRef]

35. Reid, L.M.; Nicol, R.W.; Ouellet, T.; Savard, M.; Miller, J.D.; Young, J.C.; Stewart, D.W.; Schaafsma, A.W. Interaction of Fusarium graminearum and F. moniliforme in maize ears: Disease progress, fungal biomass, and mycotoxin accumulation. Phytopathology 1999, 89, 1028-1037. [CrossRef] [PubMed]

36. Bacon, C.W.; Hinton, D.M. Symptomless endophytic colonization of maize by Fusarium moniliforme. Can. J. Bot. 1996, 74, 1195-1202. [CrossRef]

37. Foley, D.C. Systemic infection of corn by Fusarium moniliforme. Phytopathology 1962, 52, 870-872. 
38. Picot, A.; Barreau, C.; Pinson-Gadais, L.; Piraux, F.; Caron, D.; Lannou, C.; Richard-Forget, F. The dent stage of maize kernels is the most conducive for fumonisin biosynthesis under field conditions. Appl. Environ. Microbiol. 2011, 77, 8382-8390. [CrossRef]

39. Velluti, A.; Marın, S.; Bettucci, L.; Ramos, A.J.; Sanchis, V. The effect of fungal competition on colonization of maize grain by Fusarium moniliforme, F. proliferatum and F. graminearum and on fumonisin B 1 and zearalenone formation. Int. J. Food Microbiol. 2000, 59, 59-66. [CrossRef]

40. Idaho State Dept of Agriculture. Agricultural Facts. 2016. Available online: http://www.agri.idaho.gov/agri/ Categories/Marketing/Documents/2016AgStats.pdf (accessed on 24 May 2017).

41. Datnoff, L.E.; Elmer, W.H.; Huber, D.M. Mineral Nutrition and Plant Disease; APS Press: St. Paul, MN, USA, 2007; p. 278.

42. Jensen, C.A.; Olshausen, B.A. Soil Survey of Boise Area, Idaho; US Government Printing Office: Washington, DC, USA, 1901.

43. Headrick, J.M.; Pataky, J.K.; Juvik, J.A. Relationships among carbohydrate content of kernels, condition of silks after pollination, and the response of sweet corn inbred lines to infection of kernels by Fusarium moniliforme. Phytopathology 1990, 80, 487-494. [CrossRef]

44. Wilson, D.O., Jr.; Mohan, S.K. Unique seed quality problems of sh2 sweet corn. Seed Technol. 1998, 20, 176-186.

45. Duncan, K.E.; Howard, R.J. Biology of maize kernel infection by Fusarium verticillioides. Mol. Plant Microbe Interact. 2010, 23, 6-16. [CrossRef]

46. Wilke, A.L.; Bronson, C.R.; Tomas, A.; Munkvold, G.P. Seed transmission of Fusarium verticillioides in maize plants grown under three different temperature regimes. Plant Dis. 2007, 91, 1109-1115. [CrossRef]

47. Bacon, C.W.; Hinton, D.M. Endophytic and biological control potential of Bacillus mojavensis and related species. Biol. Control 2002, 23, 274-284. [CrossRef]

48. Ridout, M.E.; Newcombe, G.; Godfrey, B. First report of Fusarium temperatum in biseased sweet corn ears in the western United States. Plant Dis. 2016, 100, 2527. [CrossRef]

49. Aytoun, R.S. The effects of griseofulvin on certain phytopathogenic fungi. Ann. Bot. 1956, 20, $297-305$. [CrossRef]

50. Brian, P.W. The use of antibiotics for control of plant diseases caused by bacteria and fungi. J. Appl. Bacteriol. 1954, 17, 142-151. [CrossRef]

51. Brian, P.W.; Wright, J.M.; Stubbs, J.; Way, A.M. Uptake of antibiotic metabolites of soil micro-organisms by plants. Nature 1951, 167, 347-349. [CrossRef]

52. Crowdy, S.H.; Grovem, J.F.; Hemming, H.G.; Robinson, K.C. The translocation of antibiotics in higher plants: II. The movement of griseofulvin in broad bean and tomato. J. Exp. Bot. 1956, 7, 42-64. [CrossRef]

53. Stokes, A. Uptake and translocation of griseofulvin by wheat seedlings. Plant Soil 1954, 5, 132-142. [CrossRef]

54. Heleno, S.A.; Stojković, D.; Barros, L.; Glamočlija, J.; Soković, M.; Martins, A.; Queiroz, M.J.R.; Ferreira, I.C. A comparative study of chemical composition, antioxidant and antimicrobial properties of Morchella esculenta (L.) Pers. from Portugal and Serbia. Food Res. Internat. 2013, 51, 236-243. [CrossRef]

55. Tietel, Z.; Masaphy, S. True morels (Morchella)—Nutritional and phytochemical composition, health benefits and flavor: A review. Crit. Rev. Food Sci. Nutr. 2017, 58, 1888-1901. [CrossRef]

56. Turkoglu, A.; Kivrak, I.; Mercan, N.; Duru, M.E.; Gezer, K.; Turkoglu, H. Antioxidant and antimicrobial activities of Morchella conica Pers. Afr. J. Biotechnol. 2006, 5, 1146-1150.

57. Mohan, S.K.; Wilson, D.O. Fungi associated with seed and seedling blight of shrunken2 sweet corn in Idaho. Phytopathology 1990, 80, 891.

58. Sutton, J.C.; Baliko, W.; Funnell, H.S. Evidence for translocation of zearalenone in corn plants colonized by Fusarium graminearum. Can. J. Plant Sci. 1976, 56, 7-12. [CrossRef]

59. Mihuta-Grimm, L.; Forster, R.L. Scab of wheat and barley in southern Idaho and evaluation of seed treatments for eradication of Fusarium spp. Plant Dis. 1989, 73, 769-771. [CrossRef]

60. Baird, R.E.; Huber, D.M.; Mullinix, B.G. The mycobiota from seeds of shrunken-2 (sh2) sweet corn. Mycopathologia 1995, 132, 147-154. [CrossRef] [PubMed]

61. Wilson, D.O., Jr.; Mohan, S.K.; Knott, E.A.; Shafii, B. Evaluation of fungicide seed treatments for Shrunken-2 ("Supersweet") sweet corn. Plant Dis. 1993, 77, 348-351. [CrossRef]

62. Miller, J.D. Factors that affect the occurrence of fumonisin. Environ. Health Perspect. 2001, 109 (Suppl. 2), 321-324. 
63. Picot, A.; Barreau, C.; Pinson-Gadais, L.; Caron, D.; Lannou, C.; Richard-Forget, F. Factors of the Fusarium verticillioides-maize environment modulating fumonisin production. Crit. Rev. Microbiol. 2010, 36, 221-231. [CrossRef]

64. Campanile, G.; Ruscelli, A.; Luisi, N. Antagonistic activity of endophytic fungi towards Diplodia corticola assessed by in vitro and in planta tests. Eur. J. Plant Pathol. 2007, 117, 237-246. [CrossRef]

65. De Capdeville, G.; Wilson, C.L.; Beer, S.V.; Aist, J.R. Alternative disease control agents induce resistance to blue mold in harvested 'Red Delicious' apple fruit. Phytopathology 2002, 92, 900-908. [CrossRef]

66. Martín, J.A.; Macaya-Sanz, D.; Witzell, J. Strong in vitro antagonism by elm xylem endophytes is not accompanied by temporally stable in planta protection against a vascular pathogen under field conditions. Eur. J. Plant Pathol. 2015, 142, 185-196. [CrossRef]

67. Ridout, M.; Newcombe, G. Disease suppression in winter wheat from novel symbiosis with forest fungi. Fungal Ecol. 2016, 20, 40-48. [CrossRef]

68. European Commission Joint Research Centre; Institute for Health and Consumer Protection. Event-Specific Method for the Quantification of Maize Line NK603 Using Real-Time PCR. 2005. Available online: http://gmo-crl.jrc.ec.europa.eu/summaries/NK603-WEB-ProtocolValidation.pdf (accessed on 18 January 2017).

69. Nicolaisen, M.; Supronienè, S.; Nielsen, L.K.; Lazzaro, I.; Spliid, N.H.; Justesen, A.F. Real-time PCR for quantification of eleven individual Fusarium species in cereals. J. Microbiol. Meth. 2009, 76, 234-240. [CrossRef] [PubMed]

70. Sanger, F.; Nicklen, S.; Coulson, A.R. DNA sequencing with chain-terminating inhibitors. Proc. Natl. Acad. Sci. USA 1977, 74, 5463-5467. [CrossRef] [PubMed]

71. SAS Institute Inc. $S A S^{\circledR}$ 9.4; SAS Institute Inc.: Cary, NC, USA, 2017.

72. Zhao, S.; Fernald, R.D. Comprehensive algorithm for quantitative real-time polymerase chain reaction. $J$. Comput. Biol. 2005, 12, 1047-1064. [CrossRef]

(C) 2019 by the authors. Licensee MDPI, Basel, Switzerland. This article is an open access article distributed under the terms and conditions of the Creative Commons Attribution (CC BY) license (http://creativecommons.org/licenses/by/4.0/). 